\title{
Efectos de la densidad de cultivo y de proteína en la dieta sobre el desempeño del capaz (Pimelodus grosskopfii) en estanques
}

\author{
Effects of Dietary Culture and Protein Density on the Performance of Capaz \\ (Pimelodus grosskopfii) in Ponds
}

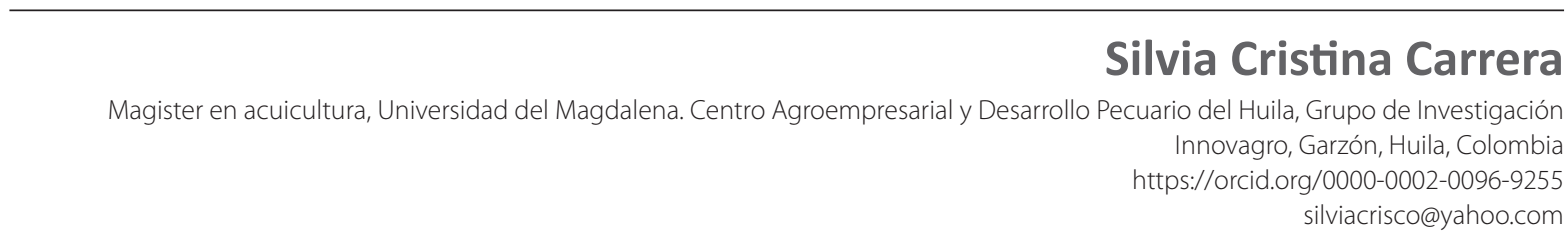

Fecha de recepción: 24 de mayo de 2019

Fecha de aceptación: 20 de septiembre de 2019

Sugerencia de citación: Carrera, S. C. (2019). Efectos de la densidad de cultivo y de proteína en la dieta sobre el desempeño del capaz (Pimelodus grosskopfii) en estanques. Mutis, 9(2), 25-33.

\section{RESUMEN}

La diversificación de la piscicultura colombiana se restringe sobre aquellas especies calificadas como promisorias. En el grupo de los silúridos se tienen representantes sobre los que se ha logrado estabilizar algún nivel de constante esfuerzo experimental en el país. En particular, con el capaz (Pimelodus grosskopfii), los desarrollos alcanzados se centran en la estandarización de manejo reproductivo, pero la información sobre su comportamiento en condiciones de cultivo aún es deficiente. En un estanque en tierra se manejaron alevinos de capaz obtenidos por reproducción artificial en tres densidades de siembra $\left(2,3\right.$ y 4 peces $\left.\mathrm{m}^{-2}\right)$, a los que se suministró concentrado comercial de 25 y 32 \% de proteína bruta (PB); se configuró un diseño con un total de 6 tratamientos, cada uno con tres réplicas. El seguimiento se realizó durante 6 meses con muestreos quincenales en los que se evaluó el desempeño. El monitoreo de calidad del agua fue constante durante el periodo. En el crecimiento se demostró una interacción significativa $(\alpha=0,05)$ entre la densidad de siembra y el porcentaje de proteína en la dieta. Se registró un mayor desempeño con $25 \%$ PB y en densidades de siembra de 2 y 4 peces $\mathrm{m}^{-2}$.

Palabras clave: P. grosskopfii, cultivo, densidad de siembra, crecimiento, estanques en tierra. 


\section{ABSTRACT}

The diversification of Colombian fish farming is restricted to those species that are described as promising. The group of the silurids includes species on whom it has been possible to stabilize some level of constant experimental effort in the country. In particular, some developments regarding the standardization of the reproductive management of able (Pimelodus grosskopfii) have been achieved; however, information on their behavior under cultivation conditions is only marginal. In a pond on the ground, fry of fodder obtained by artificial reproduction were managed at three planting densities $\left(2,3\right.$ and 4 fish $\mathrm{m}^{-2}$ ) with commercial concentrate of 25 and $32 \%$ crude protein (CP); a design was configured with a total of 6 treatments, each with three replicates. Follow-up was carried out for 6 months, with biweekly sampling in which their performance was evaluated. Water quality monitoring was constant during the period, and in the growth a significant interaction $(\alpha=0,05)$ between the seed density and the percentage of protein in the diet was demonstrated. A higher performance was recorded with $25 \% \mathrm{CP}$ and at planting densities of 2 and 4 fish $\mathrm{m}^{-2}$.

Keywords: P. grosskopfii, crop, seeding density, growth, ponds on land.

\section{INTRODUCCIÓN}

La acuicultura en Colombia se muestra como uno de los sectores de la economía que ha venido presentando un importante y significativo nivel de crecimiento anual, cuya estructura productiva depende básicamente de tres especies, dos de las cuales son consideradas exóticas (Parrado-Sanabria, 2012). A pesar de que se cuenta con suficientes opciones con potencial, la posible diversificación es un objetivo aún no materializado en la práctica. Para metas de producción en cautiverio o para establecer esquemas de repoblamiento que permitan sostener extracciones pesqueras, las limitaciones se identifican en el desconocimiento de varios aspectos de carácter biológico y técnico de algunas especies de peces (Le François, Lemieux, \& Blier, 2002). Dentro de la multiplicidad de particularidades que conlleva el término de paquete tecnológico, es claro que persisten elementos con avance muy reducidos en algunas de las especies que han sido calificadas con gran potencial productivo (Cruz et al., 2010).

El capaz (Pimelodus grosskopfii) se distribuye en las cuencas de los ríos Magdalena, Cauca, San Jorge, Sinú, Cesar, Atrato, Baudó y Catatumbo (Carrera \& Valbuena-Villareal, 2015) y en los embalses de Betania y Prado (Villaneda, 1977), ubicados en los departamentos de Huila y Tolima, respectivamente. A nivel comercial, esta especie tiene una alta aceptación y es parte importante de economías locales que se dedican a su extracción. Además, esta ha sido calificada como promisoria dentro de las posibilidades piscícolas del país (Villa-Navarro, 1999) y como parte de un desarrollo experimental que ha sido constante durante los últimos años.

Hasta el momento, se han establecido protocolos de reproducción que habilitan condiciones para asegurar una mayor oferta de alevinos, pero se reconoce que aún es necesario estandarizar procesos para que el acceso a semilla pueda alcanzar regularidad comercial (Valbuena-Villareal, Zapata, \& Cruz, 2010). La principal línea de investigación se ha enfocado en la reproducción, incluyendo algunos aspectos sobre su manejo temprano (Valbuena-Villareal, Zapata, \& Gutiérrez, 2012), hábitos alimenticios, morfometría y estados gonadales (Ramírez \& Pinilla, 2012), así como en algunos ensayos de cultivo en jaulas flotantes (Carrera \& Valbuena-Villareal, 2015). Por lo tanto, estudios publicados sobre su comportamiento en cautiverio son escasos. Por lo anterior, al igual que sucede con otros bagres, las posibilidades reales de producción piscícola comercial de esta especie siguen siendo bajas (Cruz, 2010).

Con el fin de ampliar el conocimiento y disponer de registros obtenidos directamente en condiciones de cultivo, el objetivo de este trabajo fue evaluar el desempeño productivo de juveniles de capaz en estanques en tierra bajo diferentes densidades de siembra y con varios niveles de proteína bruta en la dieta, por medio de un seguimiento adelantado durante un periodo de 6 meses. 


\section{MATERIALES Y MÉTODOS}

Los alevinos fueron adquiridos en la Estación Piscícola del Alto Magdalena, AUNAP (Gigante, Huila), y trasladados hasta una granja privada ubicada en el corregimiento de Fortalecillas (Neiva, Huila). El lote estuvo compuesto por 360 peces con un peso medio de 37,6 $\pm 2,4 \mathrm{~g}$ y una longitud total de $17,5 \pm 1,7 \mathrm{~cm}$. En un estanque de $600 \mathrm{~m}^{2}$ con divisiones internas en malla plástica se acondicionaron 18 unidades experimentales de $20 \mathrm{~m}^{2}$ cada una. Aleatoriamente, se distribuyeron tres densidades de siembra (D1:2, D2:3 y D3:4 peces $\mathrm{m}^{-2}$ ) con un suministro de concentrado comercial para tilapias con dos niveles de proteína bruta ( 25 y $32 \%)$. El ensayo se configuró con 6 tratamientos, cada uno por triplicado, para un total de 18 grupos. En el estanque, el recambio diario fue de $5 \%$ y el seguimiento tuvo una duración de 180 días.

Después de la siembra, los peces fueron mantenidos en aclimatación por 48 horas; tiempo en el que no se suministró alimento, permitiendo también que las pérdidas registradas por manejo el inicial fueran repuestas para conservar las densidades experimentales. Al no disponer de tablas de alimentación específicas, se adaptaron las tablas recomendadas por Mojica, Rodríguez y Orozco (2003) para el yaque (Leiarius marmoratus) de la siguiente manera: (i) desde la siembra hasta $30 \mathrm{~g}$ el suministro fue a voluntad; (ii) entre 30 y 150 g el 3,5\% de la biomasa; (iii) para tallas superiores, el $2,5 \%$ de la biomasa. El alimento fue ofrecido dos veces al día (9:00 y 15:00 horas) en cantidades ajustadas cada quince días con base en los muestreos de crecimiento realizados. En cada unidad, semanalmente se midieron parámetros de calidad del agua en temperatura $\left({ }^{\circ} \mathrm{C}\right)$, oxígeno disuelto $\left(\mathrm{mg} \mathrm{L}^{-1}\right)$, $\mathrm{pH}$, nitritos $\left(\mathrm{mg} \mathrm{L}^{-1}\right)$, amonio $\left(\mathrm{mg} \mathrm{L}^{-1}\right)$, alcalinidad (mg $\left.\mathrm{L}^{-1}\right)$ y dureza $\left(\mathrm{mg} \mathrm{L}^{-1}\right)$. Para el efecto se utilizó una sonda multiparamétrica (YSI 566) y un kit colorimétrico de aguas (Aquamerck).

\section{Desempeño productivo}

En los muestreos quincenales se midió el $10 \%$ de la población presente en cada unida experimental, tomando registros de longitud total $(\mathrm{cm})$, longitud estándar $(\mathrm{cm})$ y peso $(\mathrm{g})$ por medio de un ictiómetro y una balanza digital (Ohaus Scout Pro). Con los datos finales, a los 180 días se calcularon las siguientes variables productivas:

Ganancia de peso $(g): G P=$ peso final $(g)-$ peso inicial $(g)$

Ganancia en longitud $(\mathrm{cm}): G T=$ longitud final $(\mathrm{cm})-$ longitud inicial $(\mathrm{cm})$

Conversión alimenticua: $T C A=\frac{\text { Cantidad de alimento suministrado }(\mathrm{g})}{\text { ganancia total en peso }(\mathrm{g})}$

Factor de crecimiento relativo: $F C R=\frac{\text { Peso final }(\mathrm{g})}{\text { Longitud total final }(\mathrm{cm})}$

Factor de condición: $K=\frac{\text { Peso final }(\mathrm{g})}{\text { Longitud total }(\mathrm{cm}) \square^{3}}$

Tasa de crecimiento especifico: TCE $=\frac{\text { Ln peso final }- \text { Ln peso inicial }}{\text { tiempo de cultivo }} \times 100$

Sobrevivencia $\%=\frac{\mathrm{N}^{\circ} \text { final de individuos }}{\mathrm{N}^{\circ} \text { final de individuos }} \times 100$ 
En el momento de la cosecha se sacrificó el $10 \%$ de la población de cada tratamiento por sobreexposición a anestésico (MS 222). Mediante disección, se retiraron las vísceras, el hígado y la grasa presente, los cuales fueron pesados. Con los datos se calcularon los índices viscerosomático (IVS), hepatosomático (IHS) y de grasa visceral (IGV).

Los datos se presentan como media \pm desviación estándar (DE). Para determinar el efecto de los tratamientos se realizó un análisis de varianza (ANOVA) de dos vías, verificando previamente los supuestos de normalidad (prueba de Kolmogorov-Smirnov) y homogeneidad de varianzas (prueba de Levene). Cuando se presentaron, se utilizó la prueba de Tukey para precisar diferencias. El criterio de significancia fue $p<0,05$. Para un caso se estableció (por regresión) el modelo de crecimiento en peso con mayor ajuste $\left(R^{2}\right)$. Los análisis estadísticos fueron realizados con el programa SPSS 13,0.

\section{RESULTADOS}

Las variables fisicoquímicas del agua fueron relativamente estables y no se presentaron diferencias ni entre las unidades experimentales ni entre las mediciones durante el periodo de seguimiento. Con el amonio se presentó la mayor variación entre los muestreos ( $C V=84,3 \%$ ), sin que los peces llegaran a mostrar síntomas de toxicidad; los niveles de alcalinidad, dureza, con la estabilidad del pH y su cercanía a valores neutros, podrían explicar esta circunstancia (tabla 1).
A partir de la talla de siembra $(17,5 \pm 19,6 \mathrm{~cm}$ de longitud total y $37,6 \pm 2,4$ g de peso), en los tratamientos D1-25 \% y D3-25\% se alcanzaron pesos finales cercanos a los $200 \mathrm{~g}$, con incrementos en longitud y peso significativamente diferentes a los demás tratamientos (tabla 2). Se comprobó que tanto la densidad $(p=0,00)$ como el tipo de dieta $(p=0,0126)$ tuvieron una influencia significativa en la talla, con una interacción también significativa ( $p=0,002)$. Respecto a la densidad, los mejores resultados se obtuvieron en las densidades de 2 y 4 peces $\mathrm{m}^{-2}$ y alimentados con el concentrado comercial de $25 \%$ de PB. Con la combinación de 3 peces $\mathrm{m}^{-2}$ y el concentrado de $25 \% \mathrm{~PB}$, se registró la menor ganancia en peso y longitud total entre los seis tratamientos experimentales.

En cuanto al desempeño productivo, los indicadores que resultaron significativos para las densidades de 2 y 4 peces $\mathrm{m}^{-2}$ y alimentados con concentrado del 25 \% PB fueron: (i) ganancia diaria de peso, (ii) conversión alimenticia, (iii) tasa de crecimiento específica y (iv) factor de crecimiento relativo. En las variables "factor de condición" y "sobrevivencia" no se presentaron diferencias estadísticas (tabla 2). Los índices viscerosomático (IVS), hepatosomático (IHS) y de grasa visceral (IGV) calculados al finalizar el ensayo no presentaron diferencias significativas entre los 6 tratamientos evaluados (tabla 3).

Tabla 1. Valores (promedio $\pm \mathrm{DE}$ ) y coeficiente de variación (CV; \%) de los parámetros de calidad de agua en las unidades de cultivo durante el periodo experimental

\begin{tabular}{|l|c|c|c|}
\hline \multicolumn{1}{|c|}{ Parámetro } & Unidad & Valor & cV \\
\hline Temperatura & ${ }^{\circ} \mathrm{C}$ & $29 \pm 0,89$ & 3,1 \\
\hline Oxígeno disuelto & $\mathrm{mg} \mathrm{L}^{-1}$ & $4,85 \pm 0,58$ & 12,0 \\
\hline $\mathrm{pH}$ & $\mathrm{unidades}$ & $7,4 \pm 0,2$ & 2,7 \\
\hline Amonio & $\mathrm{mg} \mathrm{L}^{-1}$ & $0,23 \pm 0,197$ & 84,3 \\
\hline Nitritos & $\mathrm{mg} \mathrm{L}^{-1}$ & $0,0002 \pm 0,00004$ & 18,8 \\
\hline Alcalinidad & $\mathrm{mg} \mathrm{L}^{-1}$ & $128,25 \pm 17,93$ & 14,0 \\
\hline Dureza & $\mathrm{mg} \mathrm{L}^{-1}$ & $133,95 \pm 12,87$ & 9,6 \\
\hline
\end{tabular}


Tabla 2. Variables de desempeño productivo del capaz (P. grosskopfii) cultivado en estanques en tierra durante 6 meses

\begin{tabular}{|c|c|c|c|c|c|c|}
\hline \multirow{2}{*}{ Variables } & \multicolumn{3}{|c|}{ Concentrado comercial con $25 \%$ de PB } & \multicolumn{3}{|c|}{ Concentrado comercial con $32 \%$ de PB } \\
\hline & 2 peces $m^{-2}$ & 3 peces $m^{-2}$ & 4 peces $m^{-2}$ & 2 peces $m^{-2}$ & 3 peces $m^{-2}$ & 4 peces $m^{-2}$ \\
\hline Ganancia de peso (gr) & $159 \pm 5^{\mathrm{a}}$ & $102 \pm 12^{b}$ & $173 \pm 13^{a}$ & $112 \pm 7^{b c}$ & $130 \pm 5^{d}$ & $120 \pm 14^{b c}$ \\
\hline Ganancia de talla (cm) & $11 \pm 1^{b}$ & $9,4 \pm 0,6^{d}$ & $12 \pm 1^{a}$ & $10,4 \pm 1,4^{c}$ & $10 \pm 1^{c}$ & $11 \pm 1^{b}$ \\
\hline Ganancia diaria de peso (gr/día) & $0,88 \pm 0,01^{b}$ & $0,56 \pm 0,11^{c}$ & $0,96 \pm 0,07^{\text {a }}$ & $0,67 \pm 0,03^{c}$ & $0,72 \pm 0,02^{\mathrm{bc}}$ & $0,67 \pm 0,07$ \\
\hline Conversión alimenticia & $1,70 \pm 0,44^{\mathrm{a}}$ & $2,55 \pm 0,71^{b}$ & $1,78 \pm 0,33^{\mathrm{a}}$ & $2,36 \pm 0,17^{b}$ & $2,33 \pm 0,23^{b}$ & $2,29 \pm 0,25^{b}$ \\
\hline Tasa de crecimiento específico (\%) & $0,81 \pm 0,01^{a b}$ & $0,75 \pm 0,02^{d}$ & $0,82 \pm 0,01^{\mathrm{a}}$ & $0,78 \pm 0,09^{c d}$ & $0,78 \pm 0,07^{b c}$ & $0,78 \pm 0,06^{\mathrm{cd}}$ \\
\hline Factor de crecimiento relativo & $6,92 \pm 1,06^{\mathrm{ab}}$ & $5,17 \pm 0,17^{d}$ & $7,24 \pm 0,64^{\mathrm{a}}$ & $5,71 \pm 0,61^{\mathrm{cd}}$ & $6,18 \pm 0,33^{b c}$ & $5,66 \pm 0,19^{c d}$ \\
\hline Sobrevivencia (\%) & $95 \pm 5^{a}$ & $94 \pm 2^{a}$ & $91 \pm 6^{a}$ & $96 \pm 7^{\mathrm{a}}$ & $91 \pm 5^{\mathrm{a}}$ & $95 \pm 4^{\mathrm{a}}$ \\
\hline Factor de condición Kn & $0,0086 \pm 0,0007^{\text {a }}$ & $0,0072 \pm 0,0006^{\mathrm{a}}$ & $0,0086 \pm 0,0012^{\mathrm{a}}$ & $0,0077 \pm 0,0009^{a}$ & $0,0085 \pm 0,0007^{a}$ & $0,0072 \pm 0,0006^{a}$ \\
\hline
\end{tabular}

Nota: las letras indican diferencias significativas entre tratamientos $(p<0.05)$.

Tabla 3. Índices corporales del capaz al finalizar el seguimiento experimental cultivado en estanques en tierra durante 6 meses de cultivo

\begin{tabular}{|c|c|c|c|c|c|c|}
\hline \multirow{2}{*}{ Variables } & \multicolumn{3}{|c|}{ Concentrado comercial con $25 \%$ de PB } & \multicolumn{3}{|c|}{ Concentrado comercial con $32 \%$ de PB } \\
\hline & 2 peces $\mathrm{m}^{-2}$ & 3 peces $m^{-2}$ & 4 peces $\mathrm{m}^{-2}$ & 2 peces $m^{-2}$ & 3 peces $\mathrm{m}^{-2}$ & 4 peces $m^{-2}$ \\
\hline Índice viscerosomático & $\begin{array}{c}7,36 \pm \\
0,77^{\mathrm{a}}\end{array}$ & $8,12 \pm 0,80^{a}$ & $7,36 \pm 0,76^{a}$ & $7,53 \pm 0,17^{\mathrm{a}}$ & $7,75 \pm 1,29^{a}$ & $7,87 \pm 0,79^{a}$ \\
\hline Índice hepatosomático & $\begin{array}{c}1,05 \pm \\
0,36^{\mathrm{a}}\end{array}$ & $0,97 \pm 0,05^{a}$ & $0,88 \pm 0,14^{a}$ & $1,08 \pm 0,10^{a}$ & $1,01 \pm 0,03^{\mathrm{a}}$ & $1,00 \pm 0,07^{\mathrm{a}}$ \\
\hline Índice de grasa visceral & $\begin{array}{r}3,68 \pm \\
1,02^{a}\end{array}$ & $4,53 \pm 0,96^{a}$ & $3,82 \pm 0,49^{a}$ & $3,9 \pm 0,50^{a}$ & $3,74 \pm 0,89^{a}$ & $3,98 \pm 0,79^{a}$ \\
\hline
\end{tabular}

*Las letras indican diferencias significativas entre tratamientos $(p<0,05)$. 


\section{DISCUSIÓN}

El registro de crecimiento en la densidad evaluada de 3 peces $\mathrm{m}^{-2}$ y $25 \%$ PB no guardó consistencia con los extremos experimentales en las densidades de 2 y 4 peces $\mathrm{m}^{-2}$, lo que en principio puede ser reflejo de los menores resultados de crecimiento observados en una de las réplicas de este tratamiento; aun así, es un dato que por no tener una explicación clara debe ser objeto de validación.

Es interesante observar que fue significativa la respuesta superior en desempeño con el concentrado de menor valor de PB, lo cual coincide y confirma datos para la especie en un ensayo realizado en jaulas, cuyos resultados son similares con este mismo nivel de proteína en el alimento (Carrera \& Valbuena-Villareal, 2015).

La relación entre el peso (g) y el tiempo (días) para el tratamiento con mayor desempeño (4 peces $\mathrm{m}^{-2} \mathrm{y}$ $25 \% \mathrm{~PB})$ fue significativa $\left(R^{2}=0,976 ; p<0,001\right)$, con el mejor ajuste a un modelo de tipo exponencial para el rango de tallas trabajado. Con la ecuación definida (peso $=36,987 * \mathrm{e} 0,0102 *$ tiempo), la proyección para obtener un peso entre 250 y 300 g estaría alrededor de 6,5 meses después de la siembra, tiempo similar al que se puede proyectar con los datos obtenidos por Carrera y Valbuena-Villareal (2015) en jaulas de bajo volumen, aún con las mayores densidades evaluadas por estos autores. Se debe tener en cuenta la elevada talla inicial de los peces, por lo que a la duración del ciclo habría que adicionar el tiempo que transcurre entre un alevino de 1-2 g y los juveniles de aproximadamente $35 \mathrm{~g}$ con el que se dio comienzo al seguimiento. Se pone de relieve, entonces, que: (i) las proyecciones siguen siendo aproximadas, (ii) resulta evidente el vacío de información que se tiene en la definición de curvas de crecimiento que abarquen ciclo completo en ensayos realizados directamente bajo condiciones de cultivo comercial y (iii) la respuesta observada solo es aplicable a lo que sería manejo en levante y engorde, no extrapolable a las fases de desarrollo temprano.

La densidad de población utilizada en la actividad de acuicultura es un factor importante para el cultivo de peces debido a que densidades inadecuadas pueden conducir al estrés, lo cual afecta el comportamiento y la fisiología de los mismos (Ellis et al., 2002, Montero, Izquierdo, Tort, Robaina, \& Vergara, 1999). Sobre el desempeño de otros silúridos nativos, las referencias nacionales son escasas y los pocos reportes disponibles muestran resultados muy variables, lo que hace que las comparaciones sean limitadas y poco concluyentes hasta el momento.

Los registros que reflejan posibilidades comerciales se tienen para el yaque (Leiarius marmoratus) (Murillo-Pacheco et al., 2012), pues bajo diferentes niveles de proteína en la dieta (24, 30 y $34 \%$ PB) se alcanzan ganancias de peso entre 1,6 y $3,2 \mathrm{~g}$ día $^{-1}$ en una densidad de 1 pez $\mathrm{m}^{-2}$ y ganancias en el rango de 2,34 a 3,01 gramos día ${ }^{-1}$ en densidades de 0,5 a 2 peces $\mathrm{m}^{-2}$ (Cruz et al., 2010). En otro extremo, y si bien ha sido objeto de numerosas evaluaciones, se tiene al blanquillo (Sorubim cuspicaudus), cuyo rendimiento en sistema de jaulas apenas llega a 0,06 g de ganancia media en peso por día, según datos de Prieto, Atencio y Pardo (2005), quienes identifican algunos aspectos relacionados con la nutrición como los principales limitantes.

Bajo una estricta expectativa de cultivo que permita valorar una real competitividad o una alternativa de diversificación, los grupos que son los referentes de comparación para aguas cálidas en el país son las tilapias y las cachamas. Se tiene que, en productividad, las ganancias diarias en peso para estas especies por lo general oscilan en un rango de 2 a 4 g (Cala, Pérez, \& Rodríguez, 1996); nivel que supera al capaz, de acuerdo con lo encontrado hasta el momento. Entre los aspectos relevantes, independientemente de lo que se pueda precisar en valoraciones futuras dirigidas a mejorar estándares de desempeño para la especie, se tiene que las fortalezas del capaz se siguen puntualizando en su alta aceptación (facilidad de mercadeo) y precios comerciales favorables.

Varias consideraciones adicionales sobre los parámetros observados en este ensayo deben ser analizadas. La primera se refiere a la respuesta del capaz a un concentrado con bajo contenido de proteína. Al respecto, destaca que el factor de condición (K) que, sin diferencias entre los tratamientos, presentó una media de $0,797 \pm 0,068$, resulta ser comparable y similar al registrado de 0,805 (valor promedio entre machos y hembras), calculado en poblaciones provenientes del embalse de Betania (Cala et al., 1996). Bajo el supuesto de que en este sistema los peces acceden a fuentes de alimento que, en conjunto, cumplen sus 
requerimientos nutricionales, el valor de $\mathrm{K}$ en las condiciones de cultivo sugiere que durante el seguimiento no se manifestó alguna condición deficitaria en términos de la condición general de los peces; es decir, la relación longitud-peso en el ensayo se mantuvo dentro de límites equivalentes a los que se reportan para el medio, indicando un adecuado grado de bienestar (Ramos et al., 2013).

Si bien las exigencias nutricionales del capaz aún no están definidas, el uso de concentrados con un nivel reducido de proteína, al menos de manera preliminar, parece ser más conveniente en términos de desempeño que el de mayor contenido en PB, bajo las condiciones en las que se desarrolló este ensayo, lo que también coincide con lo registrado en jaulas (Carrera \& Valbuena-Villareal, 2015). No obstante, el hábito omnívoro con tendencia a carnívoro de la especie, la posible influencia de otras fuentes dentro de las unidades y el rango de tallas que fue trabajado, son cuestiones por valorar. En lo que se refiere al factor de conversión alimenticia, en un rango de 1,7 a 2,55 , es superior al registrado para el yaque $\left(1,51: 1 ; 1\right.$ pez $\mathrm{m}^{-2} ; 30$ \% PB) (Cruz et al., 2010) y reafirma que la línea sobre nutrición en el capaz requiere desarrollo.

El segundo factor productivo está representado en la supervivencia, que fue alta, estable y sin diferencias entre los tratamientos (promedio de 93,7 $\pm 2,1 \%$ ). La talla de los peces en el momento de la siembra fue elevada, lo que puede explicar parcialmente el resultado. Es factible también considerar que la especie muestra resistencia y adaptabilidad a condiciones de cautiverio, variable que debe ser tenida en cuenta para evaluaciones posteriores; especialmente porque permite plantear ensayos que consideren una mayor densidad en estanques. La supervivencia que reportan en jaulas (Carrera \& Valbuena-Villareal, 2015) con cargas superiores también puede ser considerada elevada (> $70 \%$ ), lo cual sugiere que las posibilidades de manejo que se tienen con capaz utilizando altas densidades pueden significar proyecciones con atractivo comercial en cultivo, tanto en estanques como en jaulas.

Por último, los peces sembrados mostraron una homogeneidad inicial relativamente alta, representada en un coeficiente de variación de 13,31 y 23,1 para la longitud total y el peso, respectivamente, lo cual fue determinado en el día 16 de seguimiento. Se observó, como rasgo especial, una disminución progresiva del $\mathrm{CV}$, con un $6,78 \%$ en la longitud y un $10,73 \%$ en peso, al final del periodo (día 180); reducción que no es usual cuando se tienen poblaciones en cautiverio, puesto que la dispersión en tallas, en este ensayo, disminuyó conforme avanzó el periodo de cultivo.

La heterogeneidad en el crecimiento depende de varios factores y su expresión es especie-específica. Aunque no es claro que necesariamente se encuentre vinculada solo al establecimiento de jerarquías sociales, también supone que intervienen otras variables con efecto en la escala que se puede presentar entre las diferencias individuales en tamaño (Baras \& Jobling, 2002; Rutten et al., 2006); se ha sugerido que en tales factores están, entre otros, la densidad de la población. Así, la disminución de la heterogeneidad inicial conforme avanza el tiempo apoyaría la hipótesis de que la especie podría llegar a tolerar densidades más elevadas de las que se contemplaron en este trabajo.

Los índices viscerosomático (IVS), hepatosomático (IHS) y de grasa visceral (IGV) no presentaron diferencia significativa entre los 6 tratamientos evaluados. Teniendo en cuenta que la longitud total de madurez (promedio entre machos y hembras) del capaz es de $27,4 \mathrm{~cm}$ (Cala, 1997), talla que se alcanzó en la media de los tratamientos en el último de los muestreos (180 días de cultivo), es posible afirmar que durante el periodo del experimento el crecimiento no se vio afectado por el desarrollo gonadal, lo cual explica los valores de los índices.

\section{CONCLUSIONES}

Con el carácter preliminar de los datos y entendiendo igualmente que existen aspectos básicos de comportamiento y manejo aún desconocidos, los registros sugieren que el capaz cuenta con interesantes posibilidades de manejo productivo en cautiverio. Los avances en la disponibilidad regular de semilla, fundamentada en protocolos ya estandarizados, es una fortaleza adicional.

El desempeño de la especie en estanques muestra parámetros con valores intermedios a los registrados para otros silúridos en el país. Los resultados, en prin- 
cipio, sustentan que sea factible inferir un válido potencial de producción con objetivos comerciales. Las limitantes en conocimiento sobre manejo en cautiverio y el carácter preliminar de estos primeros ensayos, no obstante, impiden que actualmente se puedan ofrecer proyecciones más precisas, particularmente las que involucran el comportamiento en la etapa de iniciación. Estos son elementos que deben hacer parte de líneas de investigación complementarias, considerando la ventaja que significa que tallas comerciales parecen conseguirse en tiempos razonables, aún si se comparan con los ciclos de las especies que soportan la producción piscícola en el país. Tanto la supervivencia como la condición general de los peces en el ensayo son indicativos adicionales que nos muestran que esta especie tiene gran potencial para ser utilizada comercialmente.

\section{AGRADECIMIENTOS}

La autora agradece a Colciencias (Convenio No. 1572013) por el apoyo financiero; al Centro de Desarrollo Piscícola Surcolombiano, Acuapez, y a la Universidad Surcolombiana por el apoyo logístico; a la estación piscícola Las Brisas por el espacio físico brindado para el desarrollo de este experimento.

\section{REFERENCIAS}

Baras, E., \& Jobling. M. (2002). Dynamics of intracohort canibalism in cultured fish. Aquaculture research, 33, 461-479. https://doi.org/10.1046/j.13652109.2002.00732.x

Cala, P. (1997). Espermatogénesis y ciclo anual reproductivo del capaz, Pimelodus grosskopfii (pisces: pimelodidae) en el alto río magdalena, Colombia. Caldasia, 35, 43-53.

Cala, P., Pérez, M. C., \& Rodríguez, L. (1996). Estado actual del capaz, Pimelodus grosskopfii (Pisces: Pimelodidae), en el embalse de Betania y aguas arriba en el río Magdalena, Colombia. Revista de la Academia Colombo Ciencias exactas, 20, 319-330.
Carrera, S., \& Valbuena-Villareal, R. D. (2015). Desempeño productivo del capaz (Pimelodus grosskopfii, Steindachener, 1879) bajo diferentes densidades de siembra y contenido de proteína en la dieta. Revista Intropica, 10, 20-27. https://doi. org/10.21676/23897864.1644

Cruz, N., Marciales-Caro, L. J., Díaz-Olarte, J. J., Murillo-Pacheco, R., Medina-Robles, V. M., \& Cruz-Casallas, P. E. (2010). Desempeño productivo del yaque (Leiarius marmoratus Gill, 1870) bajo diferentes densidades de siembra en estanques en tierra. Revista Colombiana de Ciencias Pecuarias, 23, 22-28.

Ellis, T., North, B., Scott, A. P., Bromage, N. R., Porter, M., \& Gaddthe, D. (2002). Relationships between stocking density and welfare in farmed rainbow trout. Journal of fish Biology, 61, 493-531. https:// doi.org/10.1111/j.1095-8649.2002.tb00893.x

Le François, N. R., Lemieux, H., \& Blier, P. U. (2002). Biological and technical evaluation of the potential of marine and anadromous fish species for cold-water mariculture. Aquaculture Research, 33, 95-108. https://doi.org/10.1046/ j.1365-2109.2002.00652.x

Mojica, H. O., Rodríguez, J. A., \& Orozco, C. R. (2003). Manual de reproducción y cultivo. El bagre rayado (Pseudoplatystoma fasciatum). Villavicencio: Inpapronatta.

Montero, D., Izquierdo, M. S., Tort, L., Robaina, L., \& Vergara J. M. (1999). High stocking density produces crowding stress altering some physiological and biochemical parameters in gilthead seabream, Sparus aurata, juveniles. Fish Physiology and Biochemistry, 20(1), 53-60. https:// doi.org/10.1023/A:1007719928905

Murillo-Pacheco, R., Cruz-Casallas, N. E., RamírezMerlano, J. A., Marciales-Caro, L. J., MedinaRobles, V. M., \& Cruz-Casallas, P. E. (2012). Efecto del nivel de proteína sobre el crecimiento del Yaque Leiarius marmoratus (Gill, 1870) bajo condiciones de cultivo. Orinoquia, 16(2), 52-61. https://doi. org/10.22579/20112629.146

Parrado-Sanabria, Y. A. (2012). Historia de la acuicultura en Colombia. Aquatic, 37, 60-77. 
Prieto, M. J., Atencio, V. J., \& Pardo, S. C. (2005). El bagre blanco Sorubim cuspicaudus y su potencial en acuicultura. Temas claves de la acuicultura. Montería: Editorial Universidad de córdoba, 70 p.

Ramírez, A., \& Pinilla. G. (2012). Hábitos alimentarios, morfometría y estados gonadales de cinco especies de peces en diferentes períodos climáticos en el río Sogamoso (Santander, Colombia). Acta Biológica Colombiana, 17(2), 241-258.

Ramos, I., Ramos, B., Heleno, S., Zanatta, A., Zica, É., Silva, R., Rezende-Ayroza, J., \& Carvalho, D. (2013). Interference of cage fish farm on diet, condition factor and numeric abundance on wild fish in a Neotropical reservoir. Aquaculture, 444, 56-62. https:// doi.org/10.1016/j.aquaculture.2013.07.013.

Rutten, M. J. M., Bovenhuis, H., Komen, J., \& Bijma, P. (2006). Mixed model methodology to infer whether aggression increases due to selection on growth in aquaculture species. In Proceedings of the 8th world congress on genetics applied to livestock production, Belo Horizonte, Brazil (pp. 9-12).

Villaneda, A. (1977). Algunos aspectos biológicos del capaz, Pimelodus grosskopfii (Trabajo de grado). Universidad Jorge Tadeo Lozano, Departamento de Biología.

Villa-Navarro, F. A. (1999). Estudio biológico pesquero de la represa de prado para la determinación de especies promisorias en acuicultura. Ibagué: Universidad del Tolima,

Valbuena-Villareal, R. D., Zapata, B., \& Cruz, P. E. (2010). Reproducción inducida de capaz (pimelodus grosskopfii) con extracto de hipófisis de carpa: reporte preliminar. Orinoquia 14(2), 133139.

Valbuena-Villareal, R. D., Zapata, B., \& Gutiérrez, M. C. (2012). Coeficientes de digestibilidad aparente de tres ingredientes proteicos para capaz, Pimelodus grosskopfii. Orinoquia 16(1), 179-186. https://doi. org/10.22579/20112629.77 